\title{
Taxpas: Epidemiological and Survival Data in Breast Cancer Patients Treated with a Docetaxel-Based Chemotherapy Regimen in South Africa
}

\author{
Shun Devan Moodley, ${ }^{1}$ Alicia McMaster, ${ }^{2}$ and Rashem Mothilal ${ }^{2}$ \\ ${ }^{1}$ The Donald Gordon Medical Centre, Johannesburg 2193, South Africa \\ ${ }^{2}$ Medical Department, Sanofi South Africa, Midrand 1682, South Africa \\ Correspondence should be addressed to Alicia McMaster; alicia.mcmaster@sanofi.com
}

Received 2 August 2013; Revised 27 September 2013; Accepted 5 November 2013

Academic Editor: Georgios Giamas

Copyright (c) 2013 Shun Devan Moodley et al. This is an open access article distributed under the Creative Commons Attribution License, which permits unrestricted use, distribution, and reproduction in any medium, provided the original work is properly cited.

\begin{abstract}
Breast cancer is the leading cancer among South African women. There is limited South African epidemiological data on triplenegative breast cancer (TNBC). Taxpas was a nonrandomized observational survey conducted in multiple centres in South Africa from April 2004 to December 2010. 1632 female patients diagnosed with breast cancer, with a median age of 51 years, were enrolled in the survey. Patients were treated on a docetaxel-based chemotherapy regimen. The objective of the study was to assess epidemiological data and survival data. The incidence of TNBC was $14 \%$. The one-year survival rate for the total cohort was $84 \%$. The one-year survival rate for patients with early stage and metastatic breast cancer was recorded as $94 \%$ and $65 \%$, respectively. Patients with TNBC stage III (all ages) and stage IV ( $\leq 50$ years) had statistically significant worse 1-year survival rate compared to N-TNBC patients of the same age and stages. Conclusion. The incidence of TNBC in South Africa which is $14 \%$ is comparable to global incidence. The 1-year survival data for certain subgroups supports the literature saying that TNBC carries a worse prognosis compared to N-TNBC. Women $\leq 50$ years diagnosed with late stage TNBC carried the worst prognosis in this survey.
\end{abstract}

\section{Introduction}

Breast cancer is the leading cancer among South African women who have a 1 in 29 lifetime risk of developing breast cancer, with an age-standardized incidence rate of 30.6 per 100,000 population [1]. Triple-negative breast cancer (TNBC) is characterized by the lack of expression of estrogen receptor $(\mathrm{ER})$, progesterone receptor (PR), and human epidermal growth factor receptor 2 (HER-2) [2]. These cancers occur in approximately 12 to $17 \%$ of women with breast cancer and are associated with an unfavourable prognosis [3].

Chemotherapy is the mainstay of advanced breast cancer treatment. Taxanes, together with anthracyclines, represent classes of drugs with very strong evidence of efficacy. Docetaxel, in particular, is an effective antineoplastic agent against both locally advanced and metastatic breast cancer and has shown increased survival rates in studies conducted in both early and advanced stage breast cancer patients irrespective of age, hormonal expression, or HER-2 status $[4,5]$.

There is limited local epidemiological data on TNBC versus N-TNBC, especially with regard to the impact of age, stage, and treatment regimens on survival. In the Taxpas survey particular attention was paid to the TNBC subgroup of patients.

\section{Methods}

Taxpas was a prospective, non-randomized, multicenter, observational survey of breast cancer patients treated with a docetaxel-based chemotherapy regimen. The survey was conducted with the voluntary assistance of private sector oncologists from 46 centers in South Africa, who had made the independent decision to prescribe docetaxel to their patients. The physician, in accordance with their practice 
TABLE 1: Number of patients according to stage.

\begin{tabular}{lcccc}
\hline & Overall $n(\%)$ & Early stage breast cancer $n(\%)$ & Metastatic breast cancer $n(\%)$ & Stage unknown $n(\%)$ \\
\hline Total cohort at baseline & $1632 / 1632(100 \%)$ & $1394 / 1632(85 \%)$ & $162 / 1632(10 \%)$ & $76 / 1632(5 \%)$ \\
Total cohort at 1-year followup & $1632 / 1632(100 \%)$ & $989 / 1632(61 \%)$ & $560 / 1632(34 \%)$ & $83 / 1632(5 \%)$ \\
TNBC & $232 / 1632(14 \%)$ & $149 / 232(64 \%)$ & $66 / 232(28 \%)$ & $17 / 232(7 \%)$ \\
N-TNBC & $1400 / 1632(86 \%)$ & $835 / 1400(60 \%)$ & $494 / 1400(35 \%)$ & $71 / 1400(5 \%)$ \\
Age $\leq 50$ years & $757 / 1632(46 \%)$ & $520 / 757(69 \%)$ & $193 / 757(25 \%)$ & $44 / 757(6 \%)$ \\
Age $>50$ years & $875 / 1632(53 \%)$ & $464 / 875(53 \%)$ & $367 / 875(42 \%)$ & $44 / 875(5 \%)$ \\
\hline
\end{tabular}

TABLE 2: One-year survival rate.

\begin{tabular}{lc}
\hline Subgroup & Number of patients (\%) \\
\hline Total cohort (1632) & $1369 / 1632(84 \%)$ \\
Early stage breast cancer (989) & $927 / 989(94 \%)$ \\
Metastatic breast cancer (560) & $364 / 560(65 \%)$ \\
Stage unknown (85) & $78 / 85(92 \%)$ \\
Triple-negative breast cancer (232) & $187 / 232(81 \%)$ \\
Nontriple-negative breast cancer (1400) & $1182 / 1400(84 \%)$ \\
\hline
\end{tabular}

and ethical considerations, prescribed the dose and duration of treatment with guidance from the registered prescribing information for docetaxel. Patients participated in the survey on a voluntary basis and gave signed informed consent in accordance with full ethical approval by the local independent ethics board.

\section{Statistical Analysis}

Data from all the participating centers in South Africa were combined and treated as one dataset for the purposes of the analysis. The statistical analysis of the survey was of a descriptive nature where continuous variables were summarized by mean, median, and standard deviation and minimum and maximum values, and discrete variables were summarized by frequencies and percentages. The descriptive statistics were calculated for the total breast cancer cohort, subgroups ( $\leq 50$ years and $>50$ years) to assess the influence of age and stage on overall survival, and TNBC and NTNBC subtypes to more closely examine the effect of type of breast cancer on overall survival. A subset analysis of the one-year survival rate according to the stage of the disease was performed on the patients. Survival rates were analyzed using the Kaplan-Meier method. Refer to Figures 1, 2, 3, 4, $5,6,7$, and 8 for the Kaplan-Meier survival graphs according to stage of disease and age. $P$ values were determined for the Kaplan-Meier curves using the logrank test. Hazard ratios for survival probability of TNBC versus N-TNBC, together with 95\% confidence interval (CI), were calculated.

All analyses were carried out on SAS, release 9.2, and run under Microsoft for a personal computer.

\section{Results}

4.1. Baseline Patient Characteristics. From April 30 2004, to December 4 2010, 1632 patients were enrolled with a median age of 51 years with ages ranging from 18 to 79 years. The staging at baseline showed that $1394(85 \%)$ patients had early stage breast cancer, and $162(10 \%)$ patients had metastatic disease. Please refer to Table 1 to view patients enrolled in the study according to the stage of their disease. The staging at the 1-year followup showed that a total of 989 (61\%) patients had early stage breast cancer and 560 (34\%) patients had metastatic disease. The presence or absence of metastases was unknown for the remaining $83(5 \%)$ patients at the 1-year followup visit.

4.2. Disease Progression and Treatment Discontinuation. 402 (24.6\%) patients experienced disease progression during the 1-year followup period of which 94 (5.8\%) patients experienced disease progression while being on treatment. $1316(80.6 \%)$ patients completed their prescribed regimen of treatment. The reasons for treatment discontinuation were adverse events $(6.4 \%)$, consent withdrawn $(2.1 \%)$, disease progression (5.7\%), death (1.9\%), lost to followup (0.5\%), and others $(2.8 \%)$.

4.3. One-Year Survival. $84 \%$ of the total cohort of patients (1369/1632) were alive at the one-year followup. $81 \%$ patients with TNBC (187/232) and 84\% patients with N-TNBC $(1182 / 1400)$ were alive at the 1-year followup visit. See Table 2 for one-year survival of different subgroups. At stage IV of the disease, TNBC had a dramatic decline in survival with only $43 \%$ (patients $\leq 50$ years) and $53 \%$ (patients $>50$ years) alive at the 1-year followup (refer Table 3).

4.4. One-Year Survival in Patients with Metastatic Disease. In the TNBC subgroup $17 \%$ of the patients (32/187) who were alive at the 1-year followup visit were known to have had metastatic disease. In the N-TNBC group 1182 patients were alive at the 1-year followup visit with $28 \%$ of these patients (332/1182) being known to have had metastatic disease. It could be suggested that the poorer 1-year survival in TNBC versus N-TNBC could not be attributable to the amount of patients with metastatic disease, as a greater percentage of $\mathrm{N}$ TNBC patients had metastatic disease.

Hazard ratios for survival of TNBC versus N-TNBC, together with 95\% confidence interval, are summarized in Table 4. The data with asterisk in Table 4 indicate the stage and age combination where the survival curves differ significantly (by the logrank test, $P<0.05$ ), with corresponding hazard ratios indicating a statistically significant worse survival probability for patients with TNBC. 
TABLE 3: One-year survival according to stage and age.

\begin{tabular}{lcccc}
\hline & TNBC $\leq 50$ years & N-TNBC $\leq 50$ years & TNBC > 50 years & N-TNBC > 50 years \\
\hline Total & $99 / 120(83 \%)$ & $571 / 637(90 \%)$ & $88 / 112(79 \%)$ & $611 / 763(80 \%)$ \\
Stage I & $15 / 17(88 \%)$ & $43 / 46(93 \%)$ & $8 / 9(89 \%)$ & $33 / 36(92 \%)$ \\
Stage II & $45 / 46(98 \%)$ & $210 / 218(96 \%)$ & $37 / 39(95 \%)$ & $211 / 225(94 \%)$ \\
Stage III & $20 / 22(91 \%)$ & $159 / 171(93 \%)$ & $13 / 16(81 \%)$ & $130 / 139(94 \%)$ \\
Stage IV & $12 / 28(43 \%)$ & $125 / 165(76 \%)$ & $20 / 38(53 \%)$ & $207 / 329(63 \%)$ \\
Stage unknown & $7 / 7(100 \%)$ & $34 / 37(92 \%)$ & $10 / 10(100 \%)$ & $30 / 34(88 \%)$ \\
\hline
\end{tabular}

TABLE 4: Statistical analyses based on survival curves: TNBC versus N-TNBC.

\begin{tabular}{lcccc}
\hline & & \multicolumn{2}{c}{$>$} & \multicolumn{2}{c}{$>$ years } \\
& Logrank $P$ value & Hazard ratio $(95 \%$ confidence interval $)$ & Logrank $P$ value & Hazard ratio \\
\hline Stage I & $P=0.440$ & $0.352(0.022-5.631)$ & $P=0.428$ & Not calculable \\
Stage II & $P=$ Not calculable & Not calculable & $P=0.555$ & $0.512(0.053-4.926)$ \\
Stage III & $P=0.017^{*}$ & $0.133(0.019-0.943)^{*}$ & $P=0.002^{*}$ & $0.138(0.031-0.619)^{*}$ \\
Stage IV & $P=0.012^{*}$ & $0.444(0.231-0.853)^{*}$ & $P=0.229$ & $0.718(0.417-1.235)$ \\
\hline
\end{tabular}

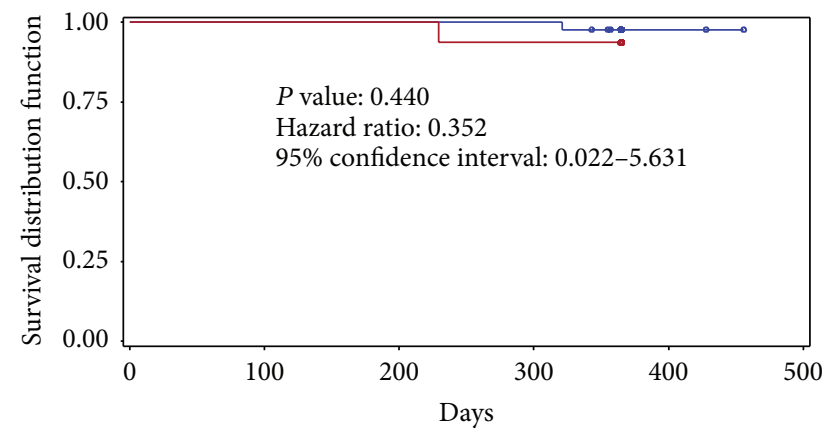

Strata:

- Group = not triple NNN

$\circ \circ \circ$ Censored group $=$ not triple NNN

- Group = triple NNN

$\circ \circ \circ$ Censored group $=$ triple $\mathrm{NNN}$

FIgURE 1: Kaplan-Meier survival graph: stage I (age $\leq 50$ years).

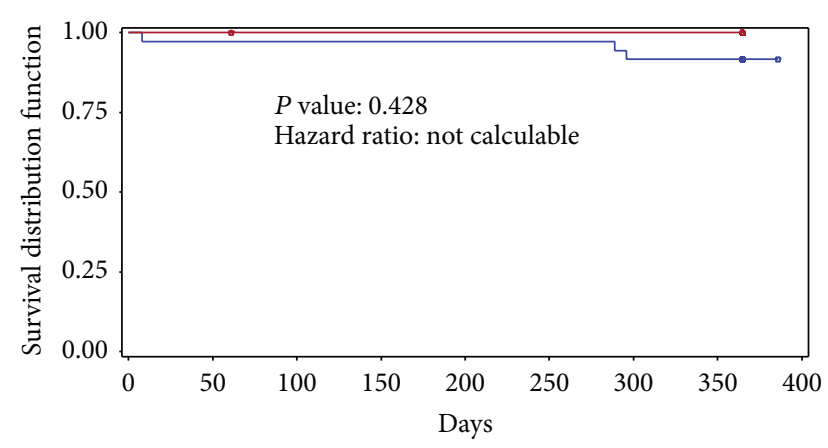

Strata:

- Group = not triple NNN

$\circ \circ \circ$ Censored group $=$ not triple NNN

- Group = triple NNN

$\circ \circ \circ$ Censored group $=$ triple $\mathrm{NNN}$

FIGURE 2: Kaplan-Meier survival graph: stage I (age > 50 years).

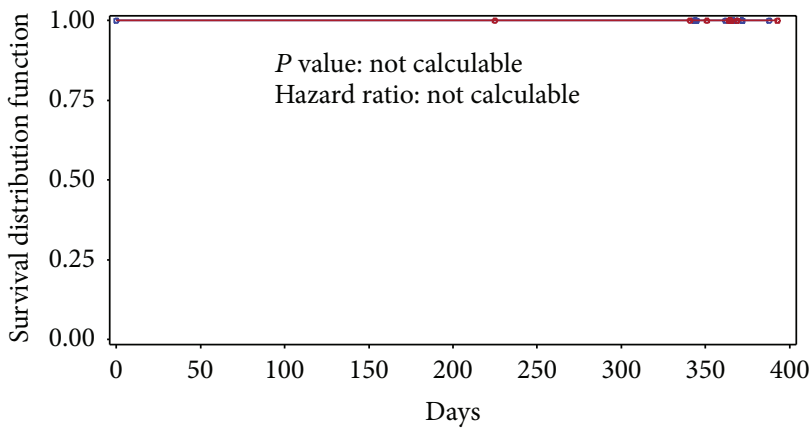

Strata:

- Group = not triple NNN

$\circ 00$ Censored group $=$ not triple NNN

- Group $=$ triple NNN

$\circ \circ \circ$ Censored group $=$ triple NNN

FIGURE 3: Kaplan-Meier survival graph: stage II (age $\leq 50$ years).

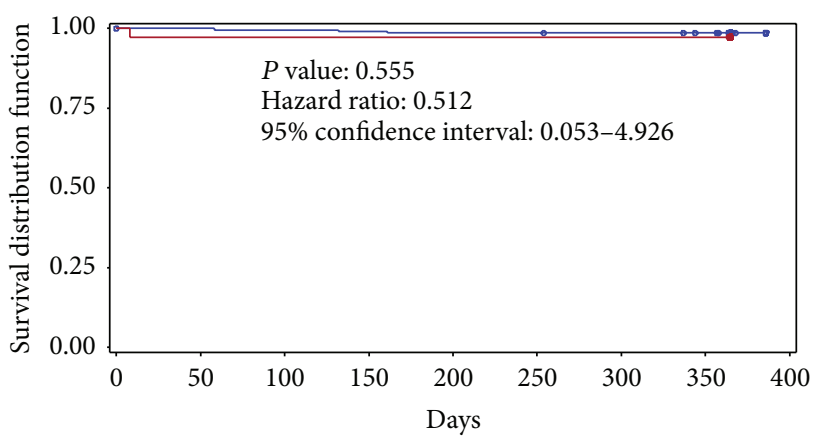

Strata:

- Group = not triple NNN

$\circ \circ \circ$ Censored group $=$ not triple $\mathrm{NNN}$

- Group = triple NNN

$\circ \circ \circ$ Censored group $=$ triple NNN

FIGURE 4: Kaplan-Meier survival graph: stage III (age > 50 years). 


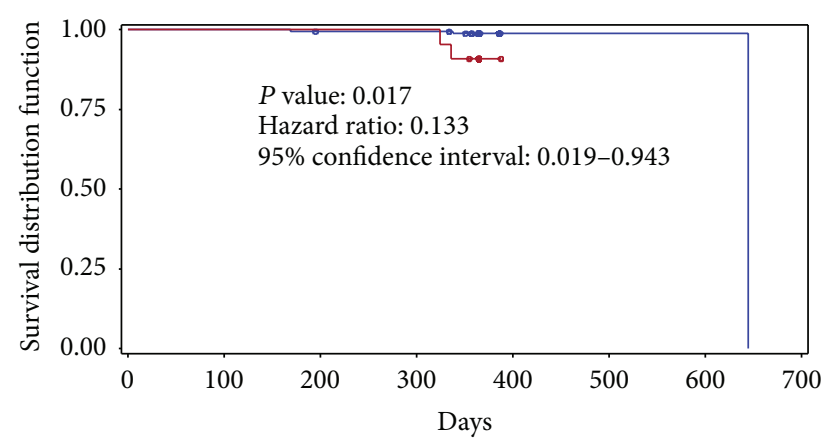

Strata:

Group $=$ not triple NNN

$\circ \circ \circ$ Censored group $=$ not triple NNN

- Group $=$ triple NNN

$\circ \circ \circ$ Censored group $=$ triple NNN

FIgURE 5: Kaplan-Meier survival graph: stage III (age $\leq 50$ years).

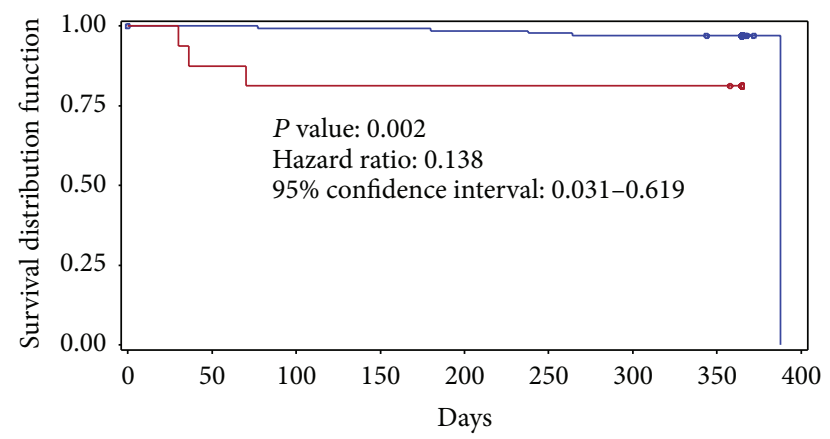

Strata:

- Group $=$ not triple NNN

$\circ \circ \circ$ Censored group $=$ not triple NNN

- Group = triple NNN

$\circ \circ \circ$ Censored group $=$ triple $\mathrm{NNN}$

FIgURE 6: Kaplan-Meier survival graph: stage III (age > 50 years).

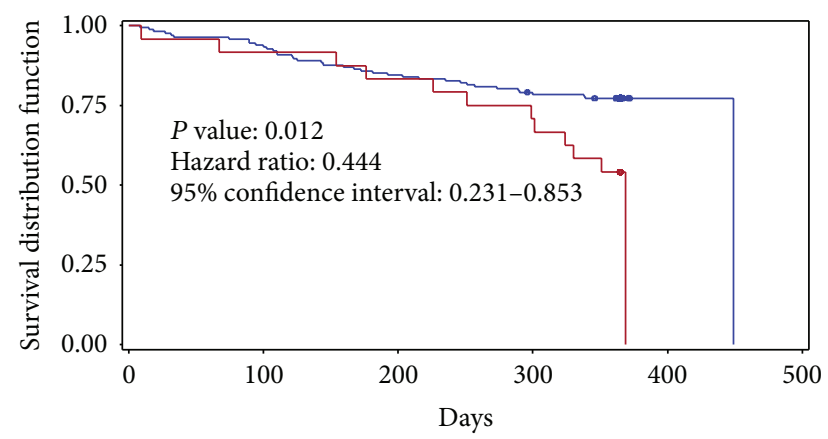

Strata:

Group = not triple NNN

○० Censored group $=$ not triple NNN

- Group = triple NNN

$\circ \circ \circ$ Censored group $=$ triple $\mathrm{NNN}$

FIGURE 7: Kaplan-Meier survival graph: stage IV (age $\leq 50$ years).
TABLE 5: Frequency of adverse events.

\begin{tabular}{lc}
\hline Adverse event & Number of patients (\%) \\
\hline Uncomplicated neutropenia & $758(46.4 \%)$ \\
Muscle cramps/spasms/myalgia & $264(16.2 \%)$ \\
Nail changes & $239(14.6 \%)$ \\
Anaemia & $153(9.4 \%)$ \\
Oral mucositis & $33(2.0 \%)$ \\
Anorexia & $29(1.8 \%)$ \\
Febrile neutropenia & $23(1.4 \%)$ \\
Neutropenic sepsis & $22(1.3 \%)$ \\
Asthenia & $7(0.4 \%)$ \\
\hline
\end{tabular}

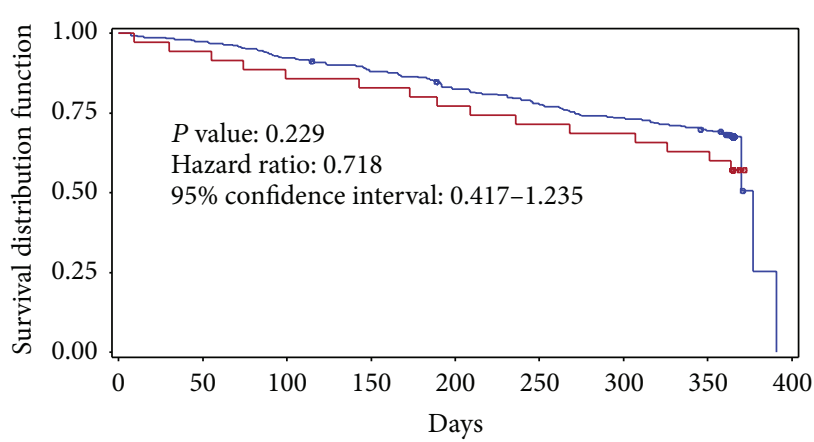

Strata:

$$
\begin{aligned}
& \text { - Group }=\text { not triple NNN } \\
& \text { ○० Censored group }=\text { not triple NNN } \\
& \text { - Group }=\text { triple NNN } \\
& \circ \circ \text { Censored group }=\text { triple NNN }
\end{aligned}
$$

FIGURE 8: Kaplan-Meier survival graph: stage IV (age $>50$ years).

4.5. Safety Data. The main recorded adverse event was uncomplicated neutropenia, which occurred in $46.4 \%$ of patients. See Table 5 for a list of adverse events and their frequencies.

\section{Discussion}

$84 \%$ of the breast cancer patients were alive at the one-year followup. The latest age-standardized relative survival rate for breast cancer in England, during 2005-2009, shows that $95.8 \%$ of women are expected to survive their disease for at least one year [6]. The 1-year survival noted in the Taxpas survey was lower than the survival reflected in the cancer survival statistics in England. This could be due to the staging of the disease at the time of diagnosis. In the Taxpas survey patients with early stage breast cancer had a 1 year survival of $94 \%$ which is comparable to the England statistics. $14 \%$ of the survey's patients had TNBC which is comparable to the global incidence [3]. The findings clearly showed that having TNBC rather than N-TNBC reduced the chances of survival by one year for all ages. This is in keeping with the current knowledge that TNBC carries a worse prognosis compared to N-TNBC, with no ability to derive benefit from molecularly targeted treatments such as endocrine therapy 
or trastuzumab, due to lack of appropriate targets for these drugs [3]. In our findings we see that survival rates were high and comparable between TNBC and N-TNBC in early disease (stages I and II), and only at stage III were marked differences seen between the two cancer types. The most dramatic decline in survival did not occur until stage IV where only $43 \%$ of TNBC patients were alive at one year. The Taxpas survey shows that this decline is most marked for patients $\leq 50$ years. Younger women are more likely to have aggressive tumours and this may be affected by genetic factors [7].

\section{Conclusion}

The findings from this survey have contributed to our understanding of the epidemiology of TNBC in South Africa which was found to be comparable to global incidence. The one-year survival rate was found to be adversely affected by metastatic disease and TNBC. The survey indicated that women $\leq 50$ years diagnosed with late stage TNBC carried the worst prognosis.

\section{Disclosure}

Dr. Shun Devan Moodley is a member of a Sanofi Advisory Board. Dr. Rashem Mothilal is the Medical Director of Sanofi South Africa. Dr. Alicia McMaster is the Medical Advisor for Oncology at Sanofi South Africa.

\section{Acknowledgments}

This work was supported by Sanofi South Africa. The medical writer is Dr. R. Moont and the statistician is Professor H.S. Schoeman.

\section{References}

[1] B. M. Wadler, C. M. Judge, M. Prout, J. D. Allen, and A. C. Geller, "Improving breast cancer control via the use of community health workers in South Africa: a critical review," Journal of Oncology, vol. 2011, Article ID 150423, 8 pages, 2011.

[2] C. Liedtke, C. Mazouni, K. R. Hess et al., "Response to neoadjuvant therapy and long-term survival in patients with triple-negative breast cancer," Journal of Clinical Oncology, vol. 26, no. 8, pp. 1275-1281, 2008.

[3] W. D. Foulkes, I. E. Smith, and J. S. Reis-Filho, "Triple-negative breast cancer," The New England Journal of Medicine, vol. 363, no. 20, pp. 1938-1948, 2010.

[4] F. Nuzzo, A. Morabito, A. Gravina et al., "Effects on quality of life of weekly docetaxel-based chemotherapy in patients with locally advanced or metastatic breast cancer: results of a singlecentre randomized phase 3 trial," BMC Cancer, vol. 11, article 75, 2011.

[5] J. P. Jacquin, S. Jones, N. Magné et al., "Docetaxel-containing adjuvant chemotherapy in patients with early stage breast cancer. Consistency of effect independent of nodal and biomarker status: a meta-analysis of 14 randomized clinical trials," Breast Cancer Research and Treatment, vol. 134, no. 3, pp. 903-913, 2012.
[6] Office for National Statistics (ONS), Cancer Survival in England: Patients Diagnosed 2005-2009 and Followed up to 2010, ONS, London, UK, 2011.

[7] J. R. Pirone, M. D’Arcy, D. A. Stewart et al., "Age-associated gene expression in normal breast tissue mirrors qualitative age-at-incidence patterns for breast cancer," Cancer Epidemiol Biomarkers Prev, vol. 21, no. 10, pp. 1735-1744, 2012. 


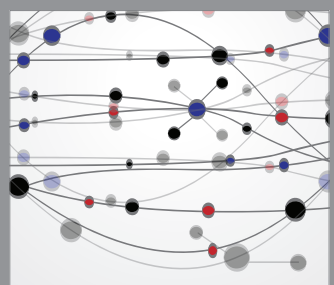

The Scientific World Journal
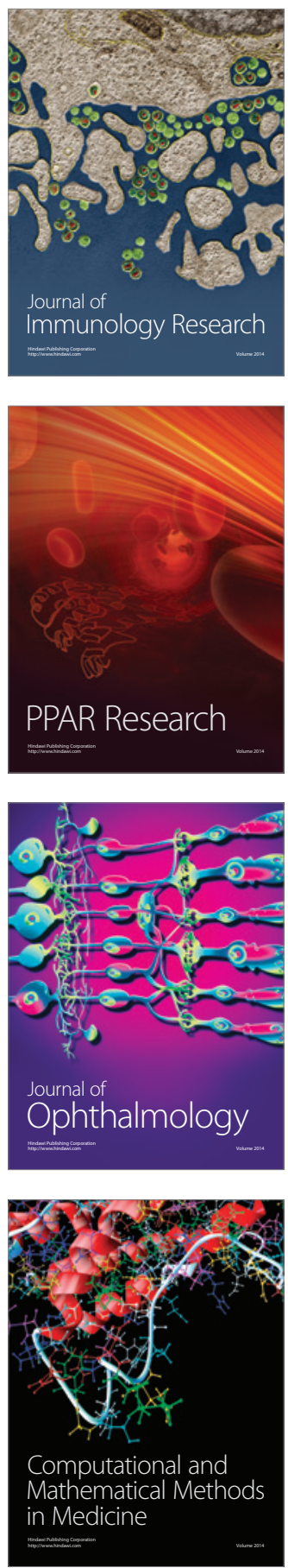

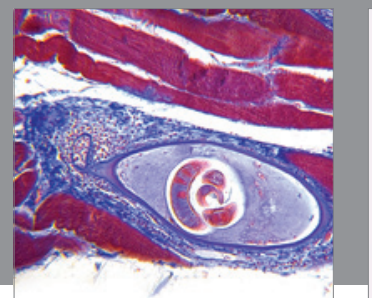

Gastroenterology

Research and Practice
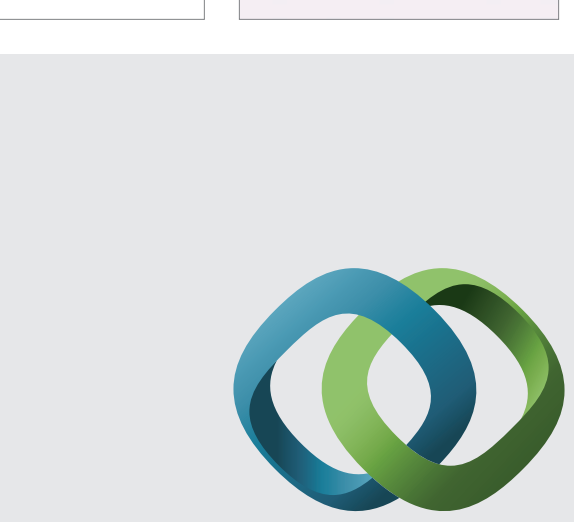

\section{Hindawi}

Submit your manuscripts at

http://www.hindawi.com
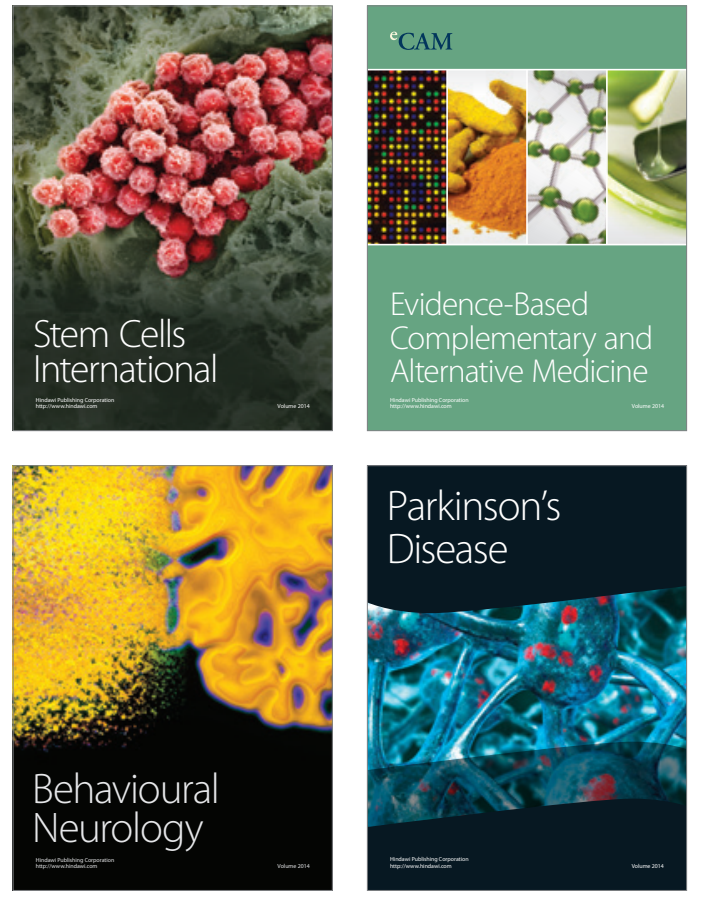
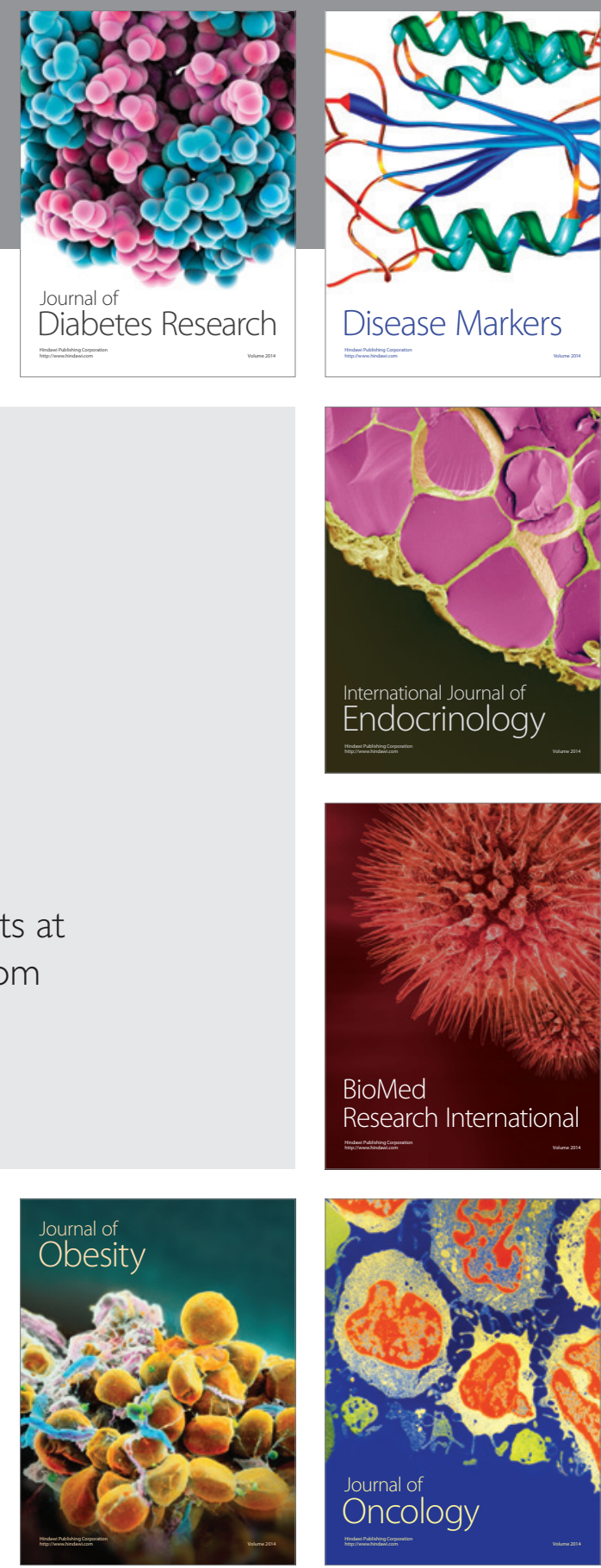

Disease Markers
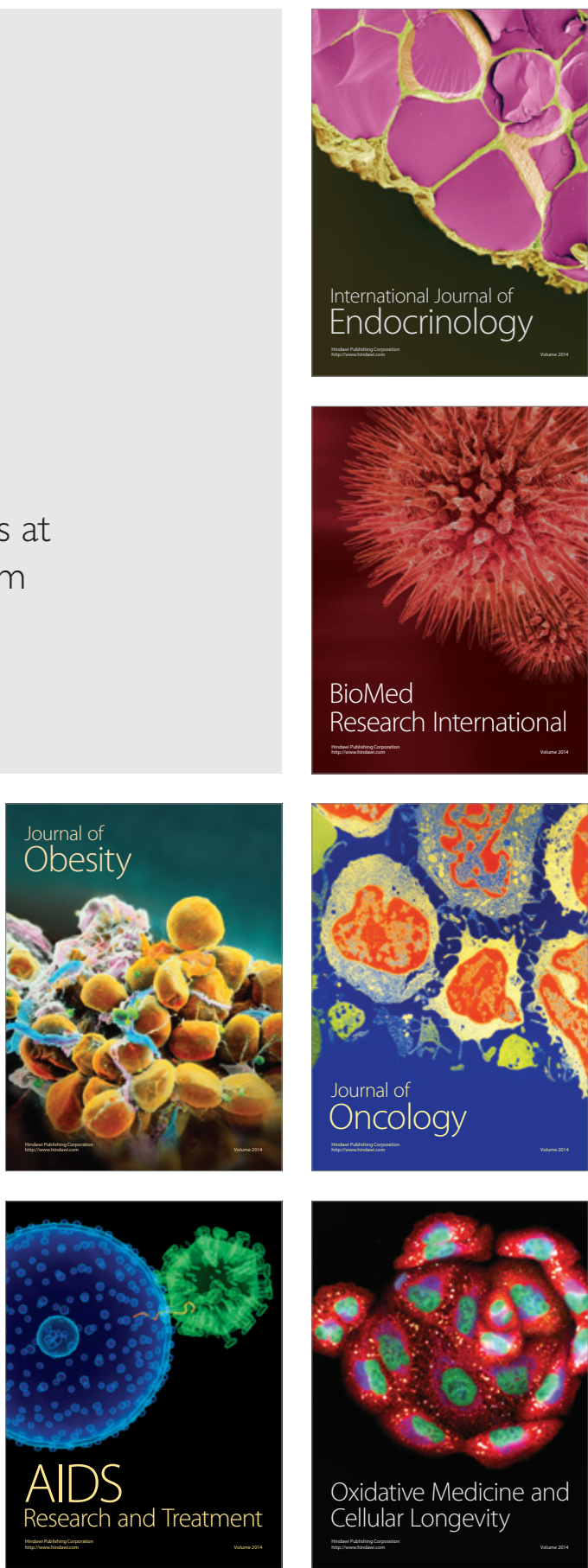